\title{
Medidas de Dispersión
}

\section{Measures of Dispersion}

\author{
Rocío B. Mayorga-Ponce ${ }^{a}$, Susana B. Reyes-Torres ${ }^{b}$, Rosa M. Baltazar-Téllez ${ }^{c}$ y Abigail \\ Martínez-Alamilla ${ }^{d}$
}

\begin{abstract}
:
Since statistics pretends to analyze and interpret numerical data, the Health Sciences make use of it and more specifically the nursing guild dedicated to research, trying to explain the behavior of important phenomena of the sample studied and the universe from which this sample was taken, besides to obtain scientifically valid data; all this with the help of dispersion measures that attempt to evaluate to what amount the data obtained differ from each other tending to move away or approach having as a reference a central value.
\end{abstract}

Keywords:

Statistics, research, nursing, analysis, dispersion

\section{Resumen:}

Puesto que la estadística pretende analizar e interpretar datos numéricos, las Ciencias de la Salud hacen uso de ella y más específicamente el gremio de enfermería dedicado a la investigación, buscando explicar el comportamiento de fenómenos importantes de la muestra estudiada y el universo de donde se tomó dicha muestra, además de obtener datos científicamente válidos; todo esto con ayuda de las medidas de dispersión que pretenden evaluar en qué medida los datos obtenidos difieren entre sí tendiendo a alejarse o acercarse teniendo como referencia a un valor central.

\section{Palabras Clave:}

Estadística, investigación, enfermería, análisis, dispersión

\section{Introducción}

En el área de la salud la estadística funge como una herramienta esencial para comprender el desarrollo y comportamiento de enfermedades a través del tiempo y el espacio. En epidemiologia por ejemplo, gracias a las herramientas estadísticas, es posible estudiar a una población e identificar un problema en ella; y por lo tanto llevar a cabo una toma de decisiones respaldadas científicamente para resolver dicha problemática con la finalidad de mejorar el proceso Salud-EnfermedadAtención.
Dentro de las herramientas estadísticas de utilidad en la investigación para la salud se encuentran las medidas de dispersión también llamadas medidas de variabilidad, comúnmente utilizadas en la estadística descriptiva y buscan señalar la diseminación que presentan las variables creando intervalos en un conjunto de datos y resumir dicha información en un solo valor tomando como punto de referencia la media aritmética (promedio). Es necesario mencionar que entre más grande sea su valor mayor será la dispersión de los datos de una distribución,

\footnotetext{
Autor de Correspondencia, Universidad Autónoma del Estado de Hidalgo, https://orcid.org/0003-3544-7171, Email: rmponce@uaeh.edu.mx

${ }^{\mathrm{b}}$ Universidad Autónoma del Estado de Hidalgo, https://orcid.org/0000-0002-9830-2589, Email: re375087@uaeh.edu.mx

c Universidad Autónoma del Estado de Hidalgo, https://orcid.org/0000-0002-2168-7564, Email: rbaltazartellez@yahoo.com.mx

d Universidad Autónoma del Estado de Hidalgo, https://orcid.org/0000-0002-4712-6351, Email: abigailmartinez5809@gmail.com
} 
lo que se traduce a una menor exactitud y más inconsistencias y/o diferencias.

\section{Resumen}

Las medidas de dispersión más utilizadas en la investigación son el rango, la varianza y la desviación estándar.

Rango: puede ser encontrado en otras bibliografías como recorrido, es la diferencia entre la puntuación mayor y la puntuación menor de un conjunto de datos. Nos deja ver que tan grande puede ser una variación o un cambio.

Varianza: es la medida de dispersión que resulta de obtener el promedio de cada una de las mediciones de un grupo de datos respecto a la media. No usa la misma unidad que los datos, sino su cuadrado; por ejemplo si los datos obtenidos se refieren al peso de un grupo de personas $(\mathrm{kg})$ la varianza lo expresará en $\mathrm{kg}^{2}$.

Desviación Estándar: también llamada desviación típica, se define como la raíz cuadrada de la varianza. Pretende regresar la medida de variabilidad a las mismas unidades que presentaban los datos originalmente y es preferida para fines descriptivos.

Tanto varianza como desviación estándar no pueden arrojar números negativos, suelen ser utilizadas para el análisis de poblaciones y muestras de tal forma que hay dos maneras de representarlas: cuando se hable de varianza muestral se simbolizara como $\mathrm{s}^{2}$ cuando se hable de varianza poblacional se representara con sigma minúscula cuadrada $\left(\sigma^{2}\right)$; si de desviación muestral estándar hablamos se simbolizara con la letra s, de igual forma con la desviación poblacional estándar se hará uso del sigma $(\sigma)$.

\section{Conclusión}

A modo de conclusión se puede decir que la estadística juega un papel importante en la enfermería basada en la evidencia y en la salud pública reconociendo el hallazgo de fenómenos que pudieran presentarse en una población, donde un análisis estadísticamente válido y confiable de los datos obtenidos daría paso a la creación de nueva evidencia científica y con ello su implementación en la práctica del cuidado enfermero. De ahí que sea necesario conocer sobre las medidas de dispersión que permiten una visión más clara de las diferencias que posee un conjunto de datos y que dependiendo de la interpretación que se les dé influyen en los resultados de investigaciones en salud. En definitiva dentro de las medidas de dispersión la desviación estándar es una de las más importantes debido a su aplicación en la estadística descriptiva, ya que sin ella no sería posible identificar el grado de error cometido solo usando la media aritmética en la lectura de los valores obtenidos (teniendo en cuenta que el valor de esta es significativa en varios aspectos de la investigación), sobre todo porque la desviación estándar pretende expresar la distorsión entre un valor esperado y el valor obtenido lo más cercano posible a la realidad al momento de su explicación.

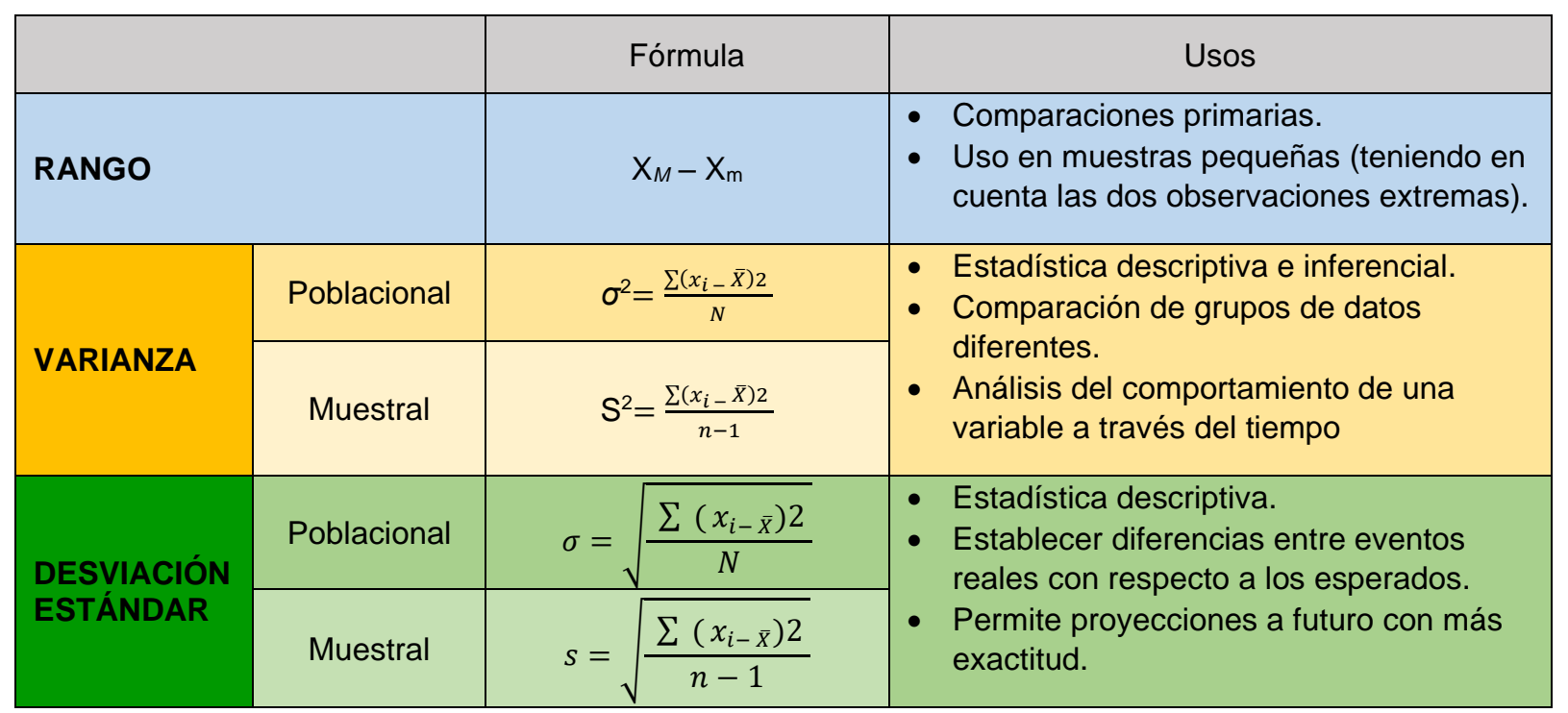

Tabla 1. Cuadro de medidas de dispersión, fórmulas para su cálculo y usos en la investigación. 


\section{Referencias}

[1] Hernández-Sampieri R., Fernández-Collado C. y Baptista-Lucio P. Metodología de la Investigación. $6^{\mathrm{a}}$ ed. México D.F.: McGRAWHILL; 2014.

[2] Mendenhall W., Beaver R y Beaver M. Introducción a la probabilidad y estadística. $14^{\mathrm{a}}$ ed. México D.F.: Cengage Learning; 2015.

[3] Álvarez-Orozco M. Unidad III Descripción análisis e interpretación de resultados [INTERNET]. Repositorio Institucional UAEM. 2019 [Citado el 10 de marzo de 2021]. Disponible en: http://ri.uaemex.mx/bitstream/handle/20.500.11799/108177/secme17262_2.pdf?sequence $=2 \&$ is Allowed $=y$

[4] Magdalena-Castro E. Bioestadística aplicada en investigación clínica: conceptos básicos. [INTERNET]. Elsevier. 2019 [Citado el 10 de marzo de 2021]. Disponible en: https://www.elsevier.es/es-revistarevista-medica-clinica-las-condes-202-articulo-bioestadisticaaplicada-investigacion-clinica-conceptos-S0716864019300045

[5] Álvarez-Aguirre A. Práctica basada en evidencia. [INTERNET].SANUS. 2019 [Citado 10 de marzo de 2021]; (6):6-7. Disponible https://sanus.unison.mx/index.php/Sanus/article/view/91

[6] Díaz P. Indicadores de salud, variables y medidas de resumen en la gestión de la salud pública. [INTERNET]. SEDICI. 2020 [Citado el 20 de marzo de 2021]. Disponible en: http://sedici.unlp.edu.ar/bitstream/handle/10915/98693/Documento_ completo.pdf-PDFA.pdf?sequence=1\&isAllowed=y

[7] Villasante P. Las medidas de dispersión en estadística. [INTERNET]. La mente es maravillosa. 2019 [Citado el 20 de marzo de 2021]. Disponible en: https://lamenteesmaravillosa.com/las-medidas-dedispersion-en-estadistica/

[8] Riquelme M. Varianza en Estadística (Uso, definición y formula). [INTERNET].Web y empresas. 2019 [Citado el 20 de marzo de 2021]. Disponible en: https://www.webyempresas.com/varianza/\#Aplicaciones_de_esta_ medida

[9] Riquelme M. ¿Qué es la Desviación Estándar o Típica? [INTERNET].Web y empresas. 2020 [Citado el 20 de marzo de 2021]. Disponible en: https://www.webyempresas.com/desviacionestandar-o-tipica/\#Usos_de_la_desviacion_estandar_o_tipica 